\title{
Layouts for Ocean Wave Energy Farms: Models, Properties, and Optimization
}

\begin{abstract}
Renewable energy, such as wave energy, plays a significant role in sustainable energy development. Wave energy represents a large untapped source of energy worldwide and potentially offers a vast source of sustainable energy. We present models and a heuristic algorithm for choosing optimal locations of wave energy conversion (WEC) devices within an array, or wave farm. The location problem can have a significant impact on the total power of the farm due to the interactions among the incident ocean waves and the scattered and radiated waves produced by the WECs. Depending on the nature of the interference (constructive or destructive) among these waves, the wave energy entering multiple devices, and thus the power output of the farm, may be significantly larger or smaller than the energy that would be seen if the devices were operating in isolation. Our algorithm chooses WEC locations to maximize the performance of a wave farm as measured by a well known performance measure called the $q$-factor, which is the ratio of the power from an array of $N$ WECs to the power from $N$ WECs operating independently, under the point absorber approximation. We prove an analytical optimal solution for the 2-WEC problem and, based on the properties of the 2-WEC solution, we propose an iterative heuristic optimization algorithm for the general problem.
\end{abstract}

\section{Introduction}

We study optimization models and algorithms for the problem of finding an optimal configuration for an array of wave energy converter (WEC) devices. Ocean wave energy represents a large untapped source of energy in the world and potentially offers a vast source of sustainable energy (Surrey and Page 1974, Wei et al. 2015). According to the Electric Power Research Institute (EPRI 2011), the total potential wave energy resource along the U.S. continental shelf edge is estimated to be 1,170 TWh per year, which is almost one third of the annual electricity consumption in the U.S. This energy resource has the advantage of being in close proximity to the coastal load centers in the U.S., which makes the transmission of the generated energy more efficient than, say, wind farms located in the geographical center of the country. Moreover, wave energy is more predictable and stable than wind and solar energy. For a summary of emerging WEC technologies, see, e.g., Kishore et al. (2013), López and Iglesias (2014). 
In the present work, we study the problem of determining the optimal layout of multiple WECs in an array, known as a wave farm. A wave farm's configuration or layout can have a significant effect on the power output of the farm, depending on the nature of the interference (constructive or destructive) among the incident ocean waves and the scattered and radiated waves produced by the WECs. In the interactions between waves and WECs or other rigid bodies, there are three types of waves (Mei 1989): Incident waves represent disturbances due to natural forces and occur whether or not the body is present; in other words, they are the incoming ocean waves. Scattered waves correspond to the collision and dispersion of incident waves with a fixed body; they represent the way in which incoming waves are deformed by the body. Finally, the motion of the body itself produces radiated waves, similar to the circular waves produced by a stone dropped into a lake. (The same three types of waves are relevant for electromagnetic waves.)

When the incident wave hits a WEC, therefore, it produces scattered and radiated waves that interact with each other and with the incident wave. Downstream WECs experience the combined wave and, in turn, produce their own scattered and radiated waves. We would like all of the WECs to be located at points of constructive interference so that they all experience waves whose amplitudes are as large as possible, thus maximizing the power produced. Unfortunately, the layout problem is complex, since the mathematical model of the hydrodynamic interactions is nonconvex and computationally expensive, and since the objective function is non separable, i.e., the power at a given WEC depends on the locations of all the other WECs.

WECs absorb mechanical power from the waves and convert it to electrical power. The conversion process is a function of how the WECs are engineered (and is often proprietary), and therefore there is no readily available function relating the mechanical power absorbed with the electrical power produced. Following the literature, therefore, we treat the absorbed mechanical power as a proxy for the electrical power produced; when we say "power," we mean mechanical power. The hydrodynamics of simple wave farms are relatively well understood, as is the absorbed power (see Section 2), but computing the exact absorbed power requires a boundary element code such as WAMIT (WAMIT, Inc. 2014). To evaluate the absorbed power for a given layout can take seconds or even minutes (for a large number of WECs and/or complex WEC geometries), and except for very special cases, a closed form analytical expression is out of the question. Therefore, exact calculations are computationally prohibitive within an optimization context. Instead, we make use of a well established approximation in our formulation, called the point-absorber approximation. In the point-absorber approximation, the devices are assumed to be small enough with respect to the wavelength of incident waves that the scattered waves can be neglected.

It is common in the literature to focus on the $q$-factor, which is the ratio between the total power 
absorbed by $N$ WECs in a wave farm to the power that would be absorbed by $N$ WECs acting in isolation:

$$
q=\frac{\sum_{n=1}^{N} P_{n}}{N P_{0}},
$$

where $P_{n}$ is the power absorbed by the $n$th device in the array and $P_{0}$ is the power absorbed by a single device acting in isolation (a constant). We follow this convention and use the $q$-factor as our objective function.

The first study related to wave farm layout goes back to Budal (1977), who investigates the theory of power absorption of a system of multiple identical interacting bodies under the assumptions of linear wave theory. ${ }^{1}$ By assuming identical bodies, one mode of motion ${ }^{2}$ and equal amplitudes, Budal (1977) simplifies the calculations using the point-absorber approximation and provides the optimal power absorption. Thomas and Evans (1981) and Falnes (1980) relax the assumption of identical motion amplitudes for oscillating devices and provide the optimal power absorption. McIver (1994) studies the hydrodynamic aspects of a system of interacting WEC devices and provides more calculations and formulations for both heaving and surging devices in an array of interacting devices.

The literature cited above provides the theoretical background for modeling the absorbed mechanical power of interacting bodies in an array. For the sake of tractability, and following the existing literature on the WEC layout problem, we consider a simple model of the ocean environment that assumes the incident waves are regular (sinusoidal) waves in water of infinite depth. We also employ the point-absorber approximation, which provides an analytical expression for the $q$-factor for a given layout. Even with these simplifying assumptions, the $q$-factor is a highly nonconvex function of the WEC locations. See Figure 1, which plots the $q$-factor vs. the location of device 1 in the 5-device layout S5A given by Fitzgerald and Thomas (2007) assuming the incident wave angle $\beta=0$; the location is plotted in Cartesian coordinates scaled by the wavenumber $k$.

From an optimization point of view, there is still a gap in the literature, since (1) the papers cited thus far evaluate the $q$-factor for a given layout but do not discuss the optimization problem of finding a layout that maximizes it, and (2) the few existing algorithms to maximize the $q$-factor appear to be relatively slow and inaccurate. In the present work, we aim to fill this gap by providing an efficient heuristic that takes advantage of the problem structure and theoretical properties.

The first attempt to optimize wave farm layouts is by Thomas and Evans (1981), who consider devices with simple geometry (e.g., spheres) laid out in simple arrangements (e.g., rows) and concludes that the spacing among devices has a larger impact on $q$ than the device geometry. Fitzgerald and Thomas

\footnotetext{
${ }^{1}$ Linear wave theory assumes that waves have small amplitude compared to their wavelength and the depth of the water, so that the hydrodynamic equations and the boundary conditions (which describe the interactions between the water and the solid WECs) can be linearized.

${ }^{2}$ For a body in water, there are three modes of translation, in the $x, y$ and $z$ directions, called surge, sway and heave, respectively. Also, there are three modes of rotation, around the $x, y$ and $z$ axes, respectively called roll, pitch and yaw.
} 


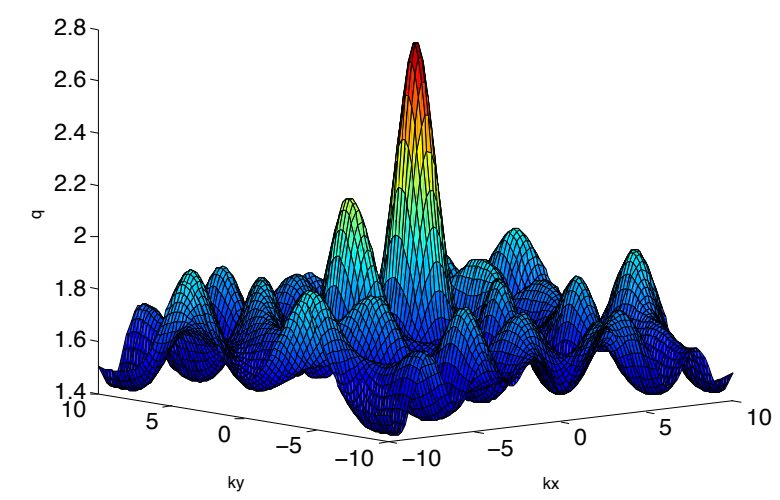

Figure 1: $q$-factor vs. Location of Device 1 in 5-WEC Layout.

(2007) appear to be the first to consider general configurations of WECs in the plane. By employing the the point-absorber approximation, they solve the 5-WEC layout optimization problem using a sequential quadratic programming (SQP) solver with multiple manually chosen starting points but do not propose a general optimization method. Our heuristic also contains a SQP-like step, but, unlike Fitzgerald and Thomas (2007), our heuristic chooses starting points systematically, based on insights gained from our analytical solution to the 2-WEC problem. Indeed, our study shows that the choice of starting point for local optimization is important. We also allow the number of devices to be general, whereas Fitzgerald and Thomas (2007) focus exclusively on the 5-WEC case.

Child (2011) and Child and Venugopal $(2009,2010)$ consider the layout problem for WECs with simplified geometries and develop mathematical equations for calculating the exact $q$-factor. They argue it is advantageous for each device to be located at the intersection points of certain parabolas centered at the other devices. They use this to develop a heuristic they call the parabolic intersection (PI) method, which they find is less accurate but faster than a genetic algorithm (GA) that they also introduce. The current work differs from theirs in the sense that we consider the point-absorber approximation rather than the exact calculation, in light of the computational difficulties discussed above. In addition, they consider both real and reactive tuning as the control mechanism for each individual device in the array, while we assume optimal tuning as is common in the literature (e.g., Budal 1977, Falnes 1980, Fitzgerald and Thomas 2007, Mavrakos and McIver 1997, McIver 1994, Thomas and Evans 1981).

Similar to our work, Mao (2013) and Snyder and Moarefdoost (2014) study the wave farm layout problem under the point-absorber approximation, and propose a "tuned" GA and a greedy search heuristic (respectively) to optimize the wave farm layout. However, unlike the heuristic algorithm in this paper, their proposed optimization algorithms do not exploit the properties of the layout problem or the structure of near-optimal solutions.

In the next section, we briefly describe the theoretical background for arrays of multiple wave energy converters. In Section 3, we formulate the optimization problem and explore its theoretical and structural 
properties. We present our heuristic in Section 4 and computational results in section 5. We discuss our conclusions in Section 6.

\section{Theoretical Background}

The total mean mechanical power absorbed by an array of $N$ identical WECs oscillating in one mode of motion, such as heave, under the standard assumptions of linear wave theory is given by (Budal 1977, Thomas and Evans 1981):

$$
P=\frac{1}{4}\left(\boldsymbol{U}^{*} \boldsymbol{X}+\boldsymbol{X}^{*} \boldsymbol{U}\right)-\frac{1}{2} \boldsymbol{U}^{*} \boldsymbol{B} \boldsymbol{U},
$$

where $\boldsymbol{U}$ is a column vector of complex velocity amplitudes (determined from the equations of motion); $\boldsymbol{X}$ is a column vector of complex exciting forces (i.e., forces acting on a floating system due to the waves) of both the incident and scattered waves; $\boldsymbol{B}$ is a matrix of real damping coefficients (i.e., parameters that quantify the reduction of oscillations in an oscillatory system); and an asterisk denotes complex conjugate transpose.

Throughout, we assume that the incident waves consist of a single sinusoid, characterized by the wave angle $\beta$ and wavenumber $k=2 \pi / \lambda$, where $\lambda$ is the wavelength. (The wavenumber is similar to frequency: Whereas frequency measures waves per unit time, wavenumber measures waves per unit distance.) Device $m \in\{1,2, \ldots, N\}$ is located at point $\left(\alpha_{m}, d_{m}\right)$ in the polar coordinate system (Figure 2$)$.

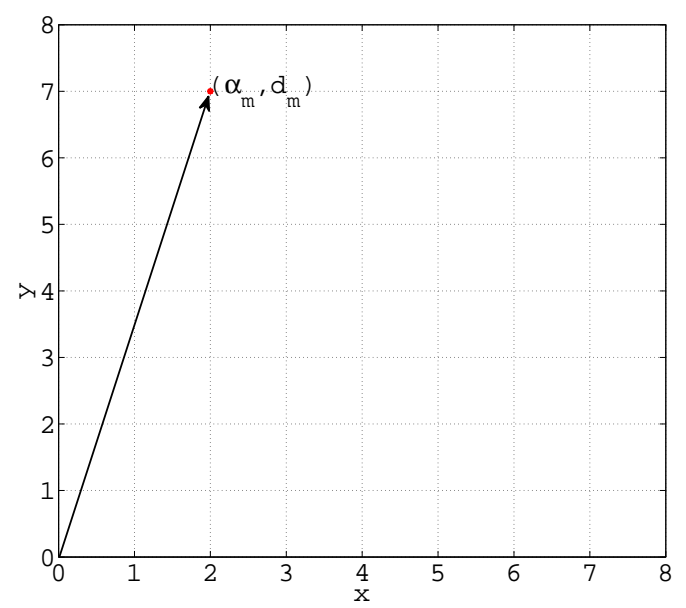

Figure 2: Location of device $m$

In (2), $\boldsymbol{U}$ is a control variable that represents the amplitude of the devices in the direction of motion. These amplitudes are realized using a braking or damping mechanism within the WEC. Not all control strategies $\boldsymbol{U}$ are attainable, due to engineering constraints on the devices, but, like most of the literature, we ignore this consideration and assume an optimal control strategy. In particular, if the WEC locations 
are fixed, then (2) may be maximized over the control variables $\boldsymbol{U}$, and the maximum power is (Thomas and Evans 1981)

$$
P_{\max }=\frac{1}{8} \boldsymbol{X}^{*} \boldsymbol{B}^{-1} \boldsymbol{X}
$$

which is attained when $\boldsymbol{U}=\frac{1}{2} \boldsymbol{B}^{-1} \boldsymbol{X}$. The expression for the power in (3) is optimized with respect to the control variables $\boldsymbol{U}$ but for fixed WEC locations. We wish to optimize the locations in order to maximize (3). Unfortunately, to calculate $P_{\max }$ in (3) requires calculating the so-called hydrodynamic coefficients $\boldsymbol{B}$ and $\boldsymbol{X}$. Since these coefficients depend on the shape, the geometry and-most relevant to our problem - the locations of the WEC devices, they must be computed numerically. As noted above, classical numerical methods are too slow to be practical within an optimization context; therefore, we employ the point-absorber approximation.

Under the point-absorber approximation, the $q$-factor in (1) has an analytical expression (Thomas and Evans 1981):

$$
q=\frac{1}{N} \boldsymbol{L}^{*} \boldsymbol{J}^{-1} \boldsymbol{L},
$$

where $\boldsymbol{L}$ is an $N$-dimensional column vector with

$$
L_{m}=e^{i k d_{m} \cos \left(\beta-\alpha_{m}\right)}
$$

and $\boldsymbol{J}$ is an $N \times N$ matrix with

$$
J_{m n}=J_{0}\left(k d_{m n}\right)
$$

$J_{0}$ is the Bessel function of the first kind with order 0 and $d_{m n}$ is the distance between device $m$ and device $n$. The advantage of (4) is that it does not involve the hydrodynamic coefficients and can thus be evaluated efficiently. Note that, although $\boldsymbol{L}$ is complex, $q$ is guaranteed to be real since $\left(\boldsymbol{L}^{*} \boldsymbol{J}^{-1} \boldsymbol{L}\right)^{*}=$ $\boldsymbol{L}^{*} \boldsymbol{J}^{-1} \boldsymbol{L}$ (since $\boldsymbol{J}$ is symmetric and real).

\section{Optimization Model}

We want to choose the locations of the WECs (in terms of $\boldsymbol{\alpha}$ and $\boldsymbol{d}$ ) in order to maximize the $q$-factor. The mathematical formulation of the Wave Energy Converter Location Problem (WECLP) is:

$$
\begin{array}{llr}
\max _{\boldsymbol{d}, \boldsymbol{\alpha}} & q(\boldsymbol{d}, \boldsymbol{\alpha} ; \beta, k)=\frac{1}{N} \boldsymbol{L}^{*} \boldsymbol{J}^{-1} \boldsymbol{L} & \\
\text { s.t. } & d_{m n} \geq d_{0} \lambda & \forall m, n=1,2, . ., N ; m \neq n \\
& \left(d_{n}, \alpha_{n}\right) \in R & \forall n=1,2, . ., N
\end{array}
$$

where $d_{0}>0$ is a constant, $\lambda$ is the incident wavelength, and $R$ is the region for locating WECs. In (7), the notation $q(d, \alpha ; \beta, k)$ indicates the $q$-factor for solution $(\boldsymbol{d}, \boldsymbol{\alpha})$ under wave angle $\beta$ and wavenumber $k$. 
We will often shorten this notation to simply $q$. The objective function depends on the decision variables $\boldsymbol{d}$ and $\boldsymbol{\alpha}$ through $\boldsymbol{L}$ and $\boldsymbol{J}$, as discussed above. Constraints (8) ensure a minimum level of separation between the devices, which reflect physical constraints and are also necessary for the point-absorber approximation to remain valid. Typically, the separation limit is a few hundred meters, large enough so that boats can maneuver among the devices for maintenance. Wavelengths in the regions where wave farms will be deployed are over 100 meters, leading to typical values of $d_{0}$ in the range of 2 or 3 . Note that the most common type of WEC currently being designed and deployed, the point absorber, has a diameter of less than 10 meters.

Proposition 1. Let $(\boldsymbol{d}, \boldsymbol{\alpha})$ be a solution to the WECLP and let $\beta$ and $k$ be a wave angle and wavenumber, respectively. Then for any wave angle $\beta^{\prime}$ and any wavenumber $k^{\prime}$, there exists a solution $\left(\boldsymbol{d}^{\prime}, \boldsymbol{\alpha}^{\prime}\right)$ such that

$$
q(\boldsymbol{d}, \boldsymbol{\alpha} ; \beta, k)=q\left(\boldsymbol{d}^{\prime}, \boldsymbol{\alpha}^{\prime} ; \beta^{\prime}, k^{\prime}\right) .
$$

Proof of Proposition 1. First suppose the wavenumber $k$ changes to $k^{\prime}$. Let $d_{n}^{\prime}=\frac{k}{k^{\prime}} d_{n}, \forall n \in$ $\{1,2, \ldots, N\}$. Since

$$
d_{m n}^{\prime}=\sqrt{d_{n}^{\prime 2}+d_{m}^{\prime 2}-2 d_{n}^{\prime} d_{m}^{\prime} \cos \left(\alpha_{m}-\alpha_{m}\right)}=\sqrt{\frac{k^{2}}{k^{\prime 2}}\left(d_{n}^{2}+d_{m}^{2}-2 d_{n} d_{m} \cos \left(\alpha_{m}-\alpha_{m}\right)\right)}=\frac{k}{k^{\prime}} d_{m n},
$$

for all $m, n \in\{1,2, \ldots, N\}$, we have $J_{0}\left(k^{\prime} d_{m n}^{\prime}\right)=J_{0}\left(k d_{m n}\right)$, and hence matrix $\boldsymbol{J}$ in (4) remains unchanged. Moreover, for all $m \in\{1,2, \ldots, N\}$, we have

$$
L_{m}^{\prime}=e^{i k^{\prime} d_{m}^{\prime} \cos \left(\beta-\alpha_{m}\right)}=e^{i k^{\prime} \frac{k}{k^{\prime}} d_{m} \cos \left(\beta-\alpha_{m}\right)}=L_{m},
$$

which means that vector $\boldsymbol{L}$ in (4) remains unchanged, as well. Therefore, we have

$$
q(\boldsymbol{d}, \boldsymbol{\alpha} ; \beta, k)=q\left(\frac{k}{k^{\prime}} \boldsymbol{d}, \boldsymbol{\alpha} ; \beta, k^{\prime}\right),
$$

as desired. Similarly, one can show that

$$
q(\boldsymbol{d}, \boldsymbol{\alpha} ; \beta, k)=q\left(\boldsymbol{d}, \boldsymbol{\alpha}+\left(\beta^{\prime}-\beta\right) \mathbf{1} ; \beta^{\prime}, k\right),
$$

where $\mathbf{1}$ is a vector of all ones.

Proposition 2. If we translate the layout along either or both axes, q remains unchanged.

Proof of Proposition 2 For all $n \in\{1,2, \ldots, N\}$, we know that

$$
x_{n}=d_{n} \cos \left(\alpha_{n}\right)
$$




$$
y_{n}=d_{n} \sin \left(\alpha_{n}\right)
$$

Thus we can write (5) as:

$$
L_{n}=e^{i k\left(x_{n} \cos (\beta)+y_{n} \sin (\beta)\right)},
$$

and for all $n, m \in\{1,2, \ldots, N\}$, we can write $d_{m n}$ in (6) as:

$$
d_{m n}=\sqrt{\left(x_{n}-x_{m}\right)^{2}+\left(y_{n}-y_{m}\right)^{2}} .
$$

In addition, let $J_{m n}^{-1}$ be the element in row $m$ and column $n$ of matrix $\boldsymbol{J}^{-1}$. Then we can expand (4) as:

$$
q=\frac{1}{N} \sum_{n=1}^{N} J_{n n}^{-1}+\frac{1}{N} \sum_{n=1}^{N-1} \sum_{m=n+1}^{N} 2 J_{n m}^{-1} \cos \left(z_{n m}\right),
$$

where $z_{n m}=k\left[\left(x_{n}-x_{m}\right) \cos (\beta)+\left(y_{n}-y_{m}\right) \sin (\beta)\right]$. Now, consider a translation along the 2-dimensional vector $(u, v)$, i.e., $\forall n \in\{1,2, \ldots, N\}$,

$$
\begin{aligned}
& x_{n}^{\prime}=x_{n}+u \\
& y_{n}^{\prime}=y_{n}+v .
\end{aligned}
$$

Under the Cartesian definition of $d_{m n}$, it is clear that $d_{m n}$ remains unchanged, as does $J_{n m}$ in (6), for all $n, m \in\{1,2, \ldots, N\}$. Moreover, since $z_{n m}$ in (10) remains unchanged, the $q$-factor does not change if we translate the layout along vector $(u, v)$.

Proposition 1 demonstrates that the WECLP is isomorphic with respect to $\beta$ and $k$. Therefore, an instance of the WECLP is completely specified by $N$, the number of devices.

\subsection{2-WEC Case}

In this section, we extend the results by Falnes (1980) on the analytical optimal solution for the special case of $N=2$. Falnes (1980) gives the optimal $q$-factor for two equal heaving bodies, but he does not characterize the optimal corresponding layout or provide a proof. Moreover, he does not explore the properties of the solution, which have significant implications for the optimization algorithm we present in Section 4.

Since translating the layout will not change the $q$-factor (Proposition 2), without loss of generality, we can assume WEC 1 is located at the origin and WEC 2 is located at $(d, \alpha)$. Then $\left(d_{1}, \alpha_{1}\right)=(0,0)$; $\left(d_{2}, \alpha_{2}\right)=(d, \alpha) ; d_{12}=d_{21}=d$. Constraint (8) simplifies to

$$
d \geq d_{0} \lambda
$$

Let $j_{n}$ be the $n$th local optimizer ( $\min$ or max) of the Bessel function $J_{0}(\cdot)$. Values to 4 decimal places are given in columns $1-3$ of Table 1 . 
Table 1: Local Optima of Bessel Function, and Resulting Optimal $q$ (for any $k, \beta$ ) and Optimal Locations of WEC 2 (for $k=0.2, \beta=0$ ).

\begin{tabular}{ccc|c|cccc}
\hline$n$ & $j_{n}$ & $J_{0}\left(j_{n}\right)$ & $q^{*}$ & $d^{*}$ & $\alpha^{*}$ & $x^{*}$ & $y^{*}$ \\
\hline 1 & 0.0000 & 1.0000 & $\infty$ & - & - & - & - \\
2 & 3.8317 & -0.4028 & 1.6744 & 19.1585 & -1.5708 & 0.0000 & -19.1585 \\
3 & 7.0156 & 0.3001 & 1.4288 & 35.0780 & -1.1065 & 15.7080 & -31.3644 \\
4 & 10.1735 & -0.2497 & 1.3328 & 50.8675 & -1.5708 & 0.0000 & -50.8675 \\
5 & 13.3237 & 0.2184 & 1.2794 & 66.6185 & -1.3328 & 15.7080 & -64.7401 \\
6 & 16.4706 & -0.1965 & 1.2445 & 82.3530 & -1.5708 & 0.0000 & -82.3530 \\
7 & 19.6158 & 0.1801 & 1.2196 & 98.0790 & -1.4099 & 15.7080 & -96.8130 \\
8 & 22.7601 & -0.1672 & 1.2007 & 113.8005 & -1.5708 & 0.0000 & -113.8005 \\
\hline
\end{tabular}

Theorem 1. Let $n^{*}$ be the smallest integer $n$ such that $j_{n} \geq 2 \pi d_{0}$. Assume that $d_{0} \geq 0.5$. Then the optimal solution to the 2-WEC problem, in polar coordinates, is to locate one WEC at $(0,0)$ and the other at $\left(d^{*}, \alpha^{*}\right)$, where

$$
\begin{aligned}
d^{*} & = \begin{cases}\frac{j_{n^{*}}}{k}, & \text { if }\left|J_{0}\left(k \lambda d_{0}\right)\right| \leq\left|J_{0}\left(j_{n^{*}}\right)\right| \\
\lambda d_{0}, & \text { otherwise }\end{cases} \\
\alpha^{*} & = \begin{cases}\beta-\arccos \left(\frac{\pi}{k d^{*}}\right), & \text { if } J_{0}\left(j_{n^{*}}\right) \geq 0, \\
\beta-\frac{\pi}{2}, & \text { otherwise. }\end{cases}
\end{aligned}
$$

The optimal solution attains a q-factor of

$$
q^{*}=\frac{1}{1-\left|J_{0}\left(k d^{*}\right)\right|} .
$$

Proof of Theorem 1. Let $z \equiv k d \cos (\beta-\alpha)$. Then

$$
\begin{gathered}
L=\left[\begin{array}{c}
1 \\
e^{i k d \cos (\beta-\alpha)}
\end{array}\right]=\left[\begin{array}{c}
1 \\
e^{i z}
\end{array}\right] \quad L^{*}=\left[\begin{array}{ll}
1 & e^{-i z}
\end{array}\right] \\
J=\left[\begin{array}{cc}
1 & J_{0}(k d) \\
J_{0}(k d) & 1
\end{array}\right] \quad J^{-1}=\frac{1}{1-J_{0}(k d)^{2}}\left[\begin{array}{cc}
1 & -J_{0}(k d) \\
-J_{0}(k d) & 1
\end{array}\right]
\end{gathered}
$$

since $J_{0}(0)=1$.

Therefore:

$$
\begin{aligned}
q & =\frac{1}{2} \mathbf{L}^{*} \mathbf{J}^{-1} \mathbf{L} \\
& =\frac{1}{2\left(1-J_{0}(k d)^{2}\right)}\left[\begin{array}{ll}
1 & e^{-i z}
\end{array}\right]\left[\begin{array}{cc}
1 & -J_{0}(k d) \\
-J_{0}(k d) & 1
\end{array}\right]\left[\begin{array}{c}
1 \\
e^{i z}
\end{array}\right]
\end{aligned}
$$




$$
\begin{aligned}
& =\frac{1}{2\left(1-J_{0}(k d)^{2}\right)}\left[1-J_{0}(k d) e^{-i z}-J_{0}(k d)+e^{-i z}\right]\left[\begin{array}{c}
1 \\
e^{i z}
\end{array}\right] \\
& =\frac{2-J_{0}(k d)\left(e^{-i z}+e^{i z}\right)}{2\left(1-J_{0}(k d)^{2}\right)} \\
& =\frac{2-J_{0}(k d)(2 \cos z)}{2\left(1-J_{0}(k d)^{2}\right)}
\end{aligned}
$$

since $e^{-i z}+e^{i z}=(\cos z+i \sin z)+(\cos z-i \sin z)=2 \cos z$

$$
=\frac{1-J_{0}(k d) \cos (k d \cos (\beta-\alpha))}{1-J_{0}(k d)^{2}} .
$$

Now, we can choose any $\alpha$, so we can make $\beta-\alpha$ equal any desired value in $[-\pi, \pi]$. Therefore, we can make $\cos (\beta-\alpha)$ equal any desired value in $[-1,1]$. Since $d_{0} \geq 0.5$ by assumption and $d \geq d_{0} \lambda$ by (11), $k d=2 \pi d / \lambda \geq \pi$. Therefore, for any $d$, we can choose $\alpha$ to make $\cos (k d \cos (\beta-\alpha))$ equal any desired value in $[-1,1]$.

Consider a fixed value of $d$. We consider two cases based on the sign of $J_{0}(k d)$.

Case 1: $J_{0}(k d) \geq 0$. Then $q$ is maximized when $\alpha$ is chosen such that $\cos (k d \cos (\beta-\alpha))=-1$, in which case

$$
q=\frac{1+J_{0}(k d)}{1-J_{0}(k d)^{2}}=\frac{1}{1-J_{0}(k d)} .
$$

To maximize $q$, we want $J_{0}(k d)$ to be as large as possible. Since the local maxima of $J_{0}(k d)$ become progressively smaller as $d$ increases, we want the smallest local argmax possible, which means choosing the smallest $d \geq d_{0} \lambda$ so that $k d$ is a local argmax of $J_{0}(\cdot)$, unless $J_{0}\left(k d_{0} \lambda\right)$ is already larger than $J_{0}$ of this $\operatorname{argmax}$, in which case we should set $d=d_{0} \lambda$. In other words,

$$
d^{*}= \begin{cases}\frac{j_{n^{*}}}{k}, & \text { if } J_{0}\left(j_{n^{*}}\right) \geq J_{0}\left(k d_{0} \lambda\right) \\ d_{0} \lambda, & \text { otherwise }\end{cases}
$$

where $n^{*}=2 m^{*}$ and $m^{*}$ is the smallest integer $m$ such that $j_{2 m} \geq k d_{0} \lambda$. (In other words, $n^{*}$ is the largest argmax greater than or equal to $k d_{0} \lambda$.) We want $\cos \left(k d \cos \left(\beta-\alpha^{*}\right)\right)=-1$, i.e.,

$$
\alpha^{*}=\beta-\arccos \left(\frac{\arccos (-1)}{k d}\right)=\beta-\arccos \left(\frac{\pi}{k d}\right) .
$$

Case 2: $J_{0}(k d)<0$. Then $q$ is maximized when $\alpha$ is chosen such that $\cos (k d \cos (\beta-\alpha))=1$, in which case

$$
q=\frac{1-J_{0}(k d)}{1-J_{0}(k d)^{2}}=\frac{1}{1+J_{0}(k d)} .
$$

To maximize $q$, we want $J_{0}(k d)$ to be as small as possible. Since the local minima of $J_{0}(k d)$ become progressively larger as $d$ increases, we want the smallest local argmin possible, which means choosing 


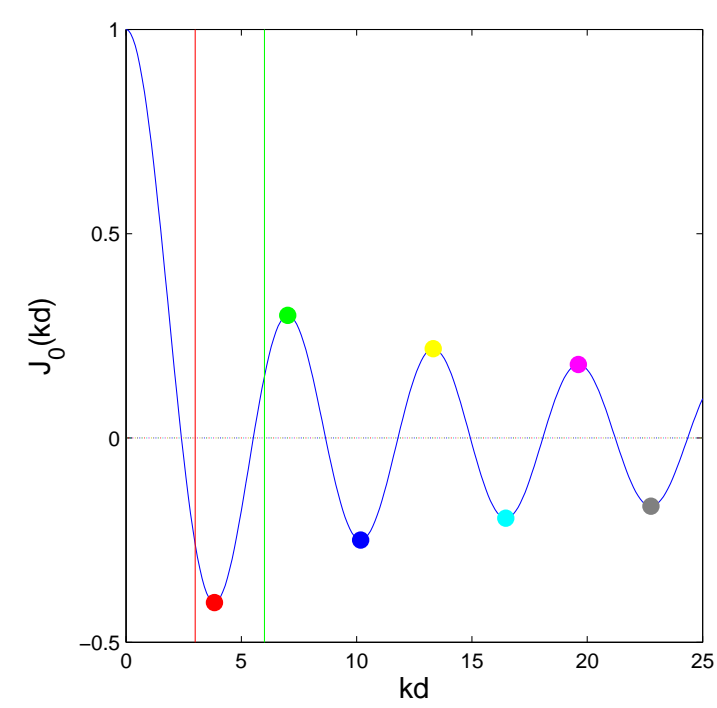

(a) Bessel Optima

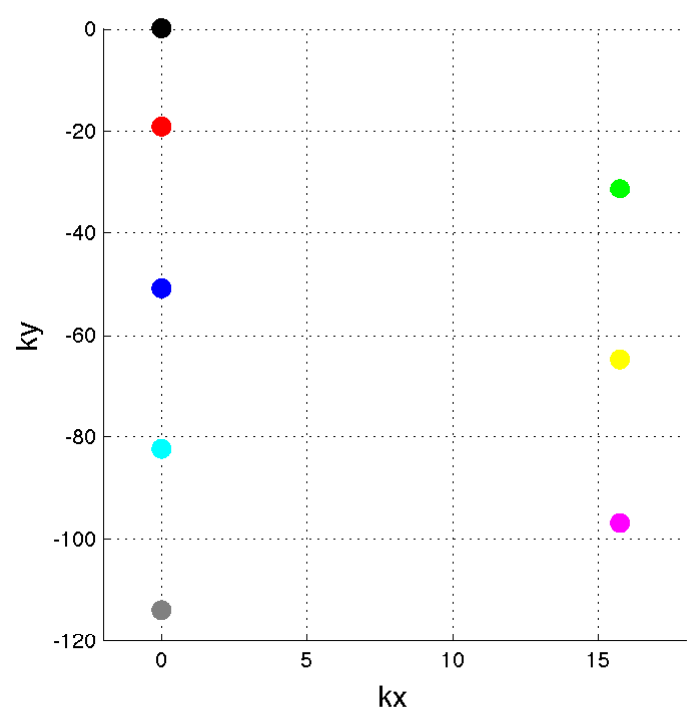

(b) Locations of WEC 2.

Figure 3: Solutions to 2-WEC Problem.

the smallest $d \geq d_{0} \lambda$ so that $k d$ is a local argmin of $J_{0}(\cdot)$, unless $J_{0}\left(k d_{0} \lambda\right)$ is already smaller than $J_{0}$ of this argmin, in which case we should set $d=d_{0} \lambda$. Therefore,

$$
d^{*}= \begin{cases}\frac{j_{n^{*}}}{k}, & \text { if } J_{0}\left(j_{n^{*}}\right) \leq J_{0}\left(k d_{0} \lambda\right) \\ d_{0} \lambda, & \text { otherwise }\end{cases}
$$

where $n^{*}=2 m^{*}+1$ and $m^{*}$ is the smallest integer $m$ such that $j_{2 m+1} \leq k d_{0} \lambda$. (In other words, $n^{*}$ is the smallest argmin greater than or equal to $k d_{0} \lambda$.) We want $\cos \left(k d \cos \left(\beta-\alpha^{*}\right)\right)=1$, i.e.,

$$
\alpha^{*}=\beta-\arccos \left(\frac{\arccos (1)}{k d}\right)=\beta-\frac{\pi}{2} .
$$

Taken together, (18) and (21) prove (12) and (17) and (20) prove (14). Finally, (19) and (22) prove (13).

Roughly speaking, Theorem 1 locates the second WEC at the local Bessel optimum (min or max) with the largest absolute value, subject to (11). Figure 3(a) displays these optima (along with hypothetical constraints (11), for the first two optima), while Figure 3(b) displays the corresponding locations of WEC 2. Column 4 of Table 1 gives the $q$-factor corresponding to each of these solutions, while columns 5-6 give the locations of WEC 2 in polar coordinates, assuming $k=0.2$ and $\beta=0$. Note that the assumption $d_{0} \geq 0.5$ in Theorem 1 is not particularly restrictive: It amounts to saying that WECs must be at least half a wavelength apart, which is completely reasonable given the need to maneuver maintenance vehicles among the WECs.

In the special case in which $\beta=0$, the Cartesian coordinates of the optimal solution are as given in the following corollary, and in Columns $7-8$ of Table 1 (assuming $k=0.2$ ). 
Corollary 1. Suppose $\beta=0$ and $d_{0} \geq 0.5$. Let $n^{*}$ be the smallest integer $n$ such that $j_{n} \geq 2 \pi d_{0}$. Then the optimal solution to the 2-WEC problem, in Cartesian coordinates, is to locate one WEC at $(0,0)$ and the other at $\left(x^{*}, y^{*}\right)$, where

$$
\begin{aligned}
& x^{*}= \begin{cases}\frac{\pi}{k}, & \text { if }\left|J_{0}\left(k \lambda d_{0}\right)\right| \leq\left|J_{0}\left(j_{n^{*}}\right)\right| \text { and } J_{0}\left(j_{n^{*}}\right) \geq 0 \\
0, & \text { otherwise }\end{cases} \\
& y^{*}= \begin{cases}-\frac{j_{n^{*}}}{k} \sqrt{1-\frac{\pi^{2}}{j_{n^{*}}^{2}}}, & \text { if }\left|J_{0}\left(k \lambda d_{0}\right)\right| \leq\left|J_{0}\left(j_{n^{*}}\right)\right| \text { and } J_{0}\left(j_{n^{*}}\right) \geq 0 \\
-\frac{j_{n^{*}}}{k}, & \text { if }\left|J_{0}\left(k \lambda d_{0}\right)\right| \leq\left|J_{0}\left(j_{n^{*}}\right)\right| \text { and } J_{0}\left(j_{n^{*}}\right)<0 \\
-\lambda d_{0}, & \text { otherwise }\end{cases}
\end{aligned}
$$

Proof of Corollary 1. If $\left|J_{0}\left(k \lambda d_{0}\right)\right|>\left|J_{0}\left(j_{n^{*}}\right)\right|$, then by Theorem $1,\left(d^{*}, \alpha^{*}\right)=\left(\lambda d_{0},-\frac{\pi}{2}\right)$. Converting to Cartesian coordinates, we have $\left(x^{*}, y^{*}\right)=\left(\lambda d_{0} \cos \left(-\frac{\pi}{2}\right), \lambda d_{0} \sin \left(-\frac{\pi}{2}\right)\right)=\left(0,-\lambda d_{0}\right)$.

Now suppose $\left|J_{0}\left(k \lambda d_{0}\right)\right| \leq\left|J_{0}\left(j_{n^{*}}\right)\right|$. If $J_{0}\left(j_{n^{*}}\right) \geq 0$, then by Case 1 in the proof of Theorem 1, $\left(d^{*}, \alpha^{*}\right)=\left(\frac{j_{n^{*}}}{k},-\arccos \left(\frac{\pi}{k d^{*}}\right)\right)$. Then

$$
\begin{aligned}
x^{*} & =d^{*} \cos \left(\alpha^{*}\right)=\frac{\pi}{k} \\
y^{*} & =d^{*} \sin \left(\alpha^{*}\right) \\
& =\frac{j_{n^{*}}}{k} \sin \left(-\arccos \left(\frac{\pi}{k d^{*}}\right)\right) \\
& =-\frac{j_{n^{*}}}{k} \sin \left(\arccos \left(\frac{\pi}{j_{n^{*}}}\right)\right) \\
& =-\frac{j_{n^{*}}}{k} \sqrt{1-\frac{\pi^{2}}{j_{n^{*}}^{2}}}
\end{aligned}
$$

using the fact that $\sin (\arccos x)=\sqrt{1-x^{2}}$. If, instead, $J_{0}\left(j_{n^{*}}\right)<0$, then by Case 2 in the proof of Theorem $1,\left(d^{*}, \alpha^{*}\right)=\left(\frac{j_{n^{*}}}{k},-\frac{\pi}{2}\right)$. Then

$$
\begin{aligned}
x^{*} & =\frac{j_{n^{*}}}{k} \cos \left(-\frac{\pi}{2}\right)=0 \\
y^{*} & =\frac{j_{n^{*}}}{k} \sin \left(-\frac{\pi}{2}\right) \\
& =-\frac{j_{n^{*}}}{k} .
\end{aligned}
$$

\section{Heuristic Algorithm}

\subsection{Structure of Near-Optimal Solutions}

In order to motivate our heuristic, we first explore the structure of optimal and near-optimal solutions. In what follows, if $d$ is a distance, then we refer to $k d$ as the non-dimensional distance. In the non- 


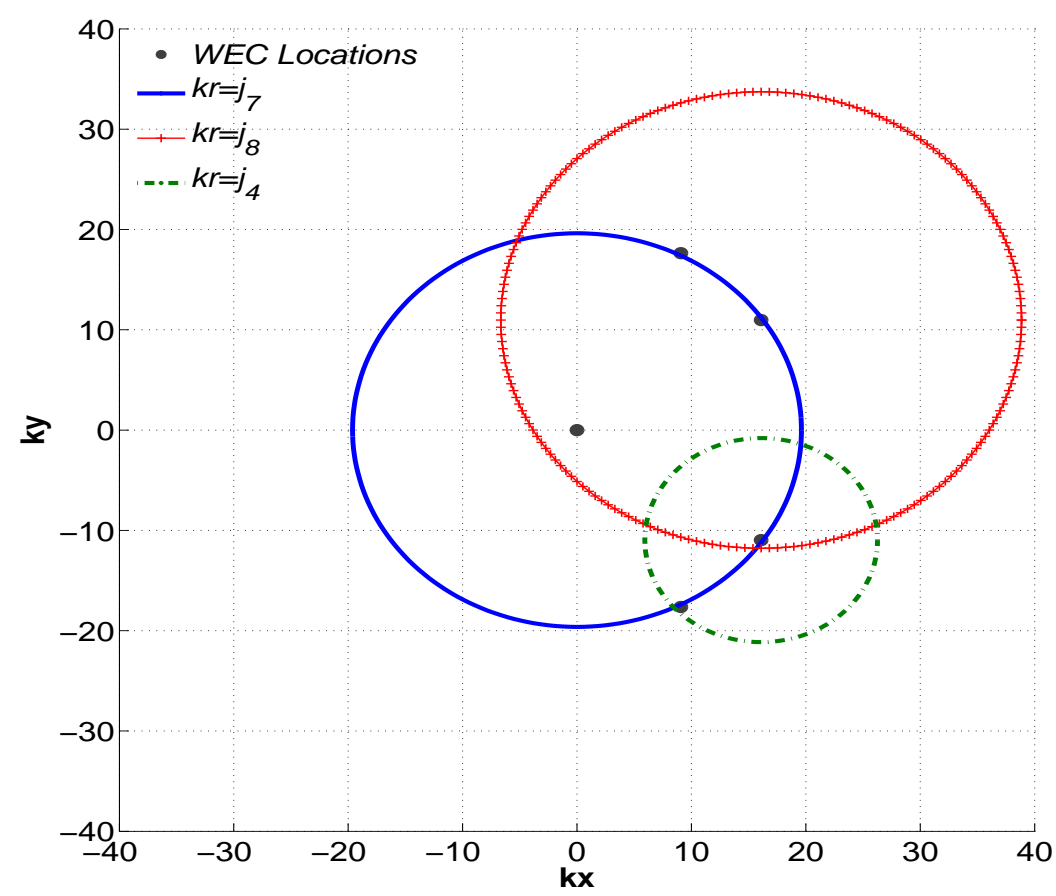

Figure 4: Pairwise Non-Dimensional Distance for the Best Known 5-WEC Layout

dimensional distance, the distance is normalized so that the unit of distance is one angular wavelength $\lambda(\lambda=\lambda / 2 \pi)$. For example, if $d=1000 \mathrm{~m}$ and $k=0.1$ (so that $\lambda=62.8 \mathrm{~m}$ and $\lambda=10$ ), then $k d=100 \mathrm{~m}=10$ angular wavelengths. We will also use the term non-dimensional layout to refer to a layout whose Cartesian coordinates have been scaled by $k$.

For the problem with $N=2$, Theorem 1 shows that the optimal non-dimensional distance between the two devices occurs at the smallest local optimizer (min or max) of the Bessel function $J_{0}(\cdot)$ that is greater than the separation limit. For larger $N$, a similar feature seems to hold approximately: Figure 4 shows the best known non-dimensional layout (from Fitzgerald and Thomas (2007)) for the case of $N=$ 5 , with $q^{*}=2.777$, assuming $\beta=0$. The circles are centered at the WECs and have radius equal to $j_{n}$ (the $n$th Bessel optimizer) for particular integer values of $n$. Note that the pairwise non-dimensional distances among the WECs are very nearly equal to the Bessel optimizers. In particular, the non-dimensional distance from WEC 1 (located at the origin) to all other WECs equals $j_{7}$. The non-dimensional distances between the remaining pairs of WECs are all very close to values in $\left\{j_{4}, j_{7}, j_{8}, j_{10}, j_{12}\right\}$. Our preliminary results suggest that a similar property holds for near-optimal solutions for other values of $N$, as well. We use this intuition to develop a heuristic algorithm for wave farms with a general number $N$ of devices. 


\subsection{Phase 1: Finding Master Layouts}

The proposed heuristic has two main phases. In the first, we search for a master layout in which the non-dimensional distance between each pair of devices equals one of the local optimizers of the Bessel function $J_{0}(\cdot)$. In the second phase, we attempt to improve the solution using a continuous local optimization procedure.

To generate master layouts, we randomly generate a matrix of pairwise non-dimensional distances that equal Bessel optimizers, and then attempt to find WEC coordinates that have this distance matrix. In particular, for each pair $m, n(m, n=1,2, \ldots, N)$, we choose the non-dimensional distance $k d_{m n}$ between $m$ and $n$ uniformly from $\left\{j_{1}, j_{2}, \ldots, j_{M}\right\}$ for $m, n=1,2, \ldots, N$ and a sufficiently large parameter $M$. (We make sure that $j_{n}$ satisfies equation (8) for all $n$, i.e., $j_{n} \geq \frac{d_{0} \lambda}{k}$.) The parameter $M$ plays an important role in the heuristic's performance, in terms of both time and accuracy. If we choose $M$ too small, the search space is reduced and it will be unlikely to find good master layouts. For example, in the best known 5-WEC layout, the maximum non-dimensional distance among the pairs of WECs approximately equals the 12th Bessel optimum, so setting $M$ equal to, say, 5 , we may not find the best layout. On the other hand, if we set $M$ to be large, the algorithm will execute more slowly. Our preliminary numerical experiments suggest that $M=2 N+3$ is a good choice.

The output of this process is an $N \times N$ distance matrix, $\boldsymbol{D}$, whose elements are the non-dimensional distances $k d_{m n}$. The next step is to find a master layout that corresponds to the distance matrix $\boldsymbol{D}$, if one exists. First, we check whether $\boldsymbol{D}$ is a Euclidean distance matrix (EDM), i.e., whether there exists a set of $N$ distinct points in the 2-dimensional Euclidean space whose pairwise distances equal the entries of the matrix $\boldsymbol{D}$. It is easy to test whether $\boldsymbol{D}$ is an EDM using the following theorem by Schoenberg (1935):

Theorem 2 (Schoenberg (1935)). A symmetric matrix $\boldsymbol{D}$ with zero diagonal and nonnegative off-diagonal elements is an EDM if and only if

$$
-\left(I-\frac{1}{N} \mathbf{1 1}^{T}\right) \boldsymbol{D}\left(\boldsymbol{I}-\frac{1}{N} \mathbf{1 1}^{T}\right) \geq 0
$$

If $\boldsymbol{D}$ is not an EDM, we simply discard it. If it is an EDM, we can find the corresponding Cartesian coordinates using methods developed in the area of Euclidean distance geometry and multidimensional scaling (Borg and Groenen 2005, Liberti et al. 2012). Consider an $N \times 2$ matrix $\mathcal{X}$ given by

$$
\boldsymbol{X}=\left(\begin{array}{c}
\boldsymbol{X}_{1}^{T} \\
\boldsymbol{X}_{2}^{T} \\
\vdots \\
\boldsymbol{X}_{N}^{T}
\end{array}\right),
$$

where $\boldsymbol{X}_{m}^{T}=\left(x_{m}, y_{m}\right)$ is the coordinates for device $m$. If $\boldsymbol{X}$ is the coordinate matrix, then we can compute 
the distance matrix $\boldsymbol{D}$ as

$$
\boldsymbol{D}^{2}(\boldsymbol{X})=\operatorname{diag}\left(\boldsymbol{X} \boldsymbol{X}^{T}\right) \mathbf{1}^{T}+\mathbf{1} \operatorname{diag}\left(\mathcal{X} \boldsymbol{X}^{T}\right)^{T}-2 \boldsymbol{X} \boldsymbol{X}^{T}
$$

Here, $\operatorname{diag}\left(\mathcal{X} \mathcal{X}^{T}\right)$ is an $N$-by-1 vector of the diagonal elements of $\mathcal{X} \boldsymbol{X}^{T}$, and $\boldsymbol{D}^{2}$ is the element-wise square of matrix $\boldsymbol{D}$. It can be proven that matrix $\boldsymbol{D}$ is invariant to transformation, rotation and reflection of $\mathcal{X}$ (Parhizkar 2013); thus, given $\boldsymbol{D}$, there are infinitely many coordinate matrices $\boldsymbol{X}$ that generate $\boldsymbol{D}$. There are several algorithms from Euclidean distance geometry and multidimensional scaling (MDS) that can be used to recover the coordinate matrix $\mathcal{X}$ from a given distance matrix $\boldsymbol{D}$. We use an algorithm called Classic Scaling to recover the corresponding coordinates (e.g., Borg and Groenen 2005); see Algorithm 1.

Algorithm 1 Classic Scaling Algorithm

1. Given $\boldsymbol{D}$, compute $\boldsymbol{D}^{2}$ by squaring elements of $\boldsymbol{D}$.

2. Compute the eigenvalues and eigenvectors of

$$
\boldsymbol{\Gamma}=-\frac{1}{2}\left(\boldsymbol{I}-\frac{1}{N} \mathbf{1 1}^{T}\right) \boldsymbol{D}^{2}\left(\boldsymbol{I}-\frac{1}{N} \mathbf{1 1}^{T}\right) .
$$

3. Choose the two largest eigenvalues and their corresponding eigenvectors. Note that the number of nonzero eigenvalues is the dimension of the solution; if there are fewer than two nonzero eigenvalues, then the layout is linear and thus easy to recover.

4. The coordinate matrix is given by:

$$
\mathcal{X}=\boldsymbol{Q}_{2} \boldsymbol{\Lambda}_{2}^{1 / 2}
$$

where $Q$ is the square $N$-by $N$ matrix whose $n$th column is the $n$th eigenvector of $\Gamma$ and $\Lambda$ is the diagonal matrix whose diagonal elements are the corresponding eigenvalues of $\boldsymbol{\Gamma}$.

\subsection{Phase 2: Local Improvement}

In the second phase of the heuristic, we take the resulting coordinates as the WEC locations in the master layout and use a continuous, convex optimization solver to attempt to improve the solution to maximize the $q$-factor. (Any convex optimization solver may be used; we used MATLAB's fminunc and fmincon functions. See additional details below.) The motivation for this phase is that the master layout found in the first phase may fall within one of the "humps" in Figure 1 but not at its peak; that is, it may fall near, but not at, a local maximum. The continuous-optimization step ensures that we reach a local maximum.

Algorithm 2 formally states the steps for our two-phase heuristic. 
0. Given: CPU time limit $T$, parameter $M$. Set $q_{\max }:=0$.

1. Randomly generate an $N \times N$ symmetric matrix whose elements equal one of the $M$ smallest local optimizers of the Bessel function $J_{0}(\cdot)$, and store it in $\boldsymbol{D}$.

2. Check whether $\boldsymbol{D}$ is an EDM using Theorem 2. If $\boldsymbol{D}$ is an EDM, go to 4, otherwise go to 1.

3. Find $x, y$ coordinates that correspond to the distance matrix $\boldsymbol{D}$ using Algorithm 1 .

4. Using the layout defined by $(x, y)$ as the initial solution, optimize all locations locally using a continuous optimization algorithm. Let $q:=$ the $q$-factor of the resulting solution.

5. Let $q_{\max }:=\max \left\{q, q_{\max }\right\}$.

6. If the elapsed CPU time $\geq T$, STOP, otherwise go to 1 .

\subsection{Symmetry}

The best known solution for $N=5$ from Fitzgerald and Thomas (2007) is symmetric with respect to a line parallel to the wave direction $\beta$. In fact, we conjecture that this property is always true of optimal solutions:

Conjecture 1. For $N \geq 2$, layouts that are symmetric with respect to the wave direction $\beta$ dominate asymmetric layouts.

If true, Conjecture 1 would imply that we can simplify the optimization problem. Thus, we can enforce symmetry using the constraints

$$
\forall n=1, \ldots, N / 2, \quad\left\{\begin{array}{l}
\alpha_{n}-\beta=\beta-\alpha_{2 n} \\
d_{n}=d_{2 n}
\end{array}\right.
$$

if $N$ is even or

$$
\forall n=1, \ldots,(N+1) / 2, \quad\left\{\begin{array}{l}
\alpha_{n}-\beta=\beta-\alpha_{2 n-1} \\
d_{n}=d_{2 n-1}
\end{array}\right.
$$

if $N$ is odd. We add these constraints to the problem solved by the solver in step 4 of Algorithm 2. (We used MATLAB's fmincon and fminunc functions for the problems with and without symmetry constraints.) In the computational results below, we refer to the problems with and without symmetry constraints as WSymC and WOSymC, respectively. 


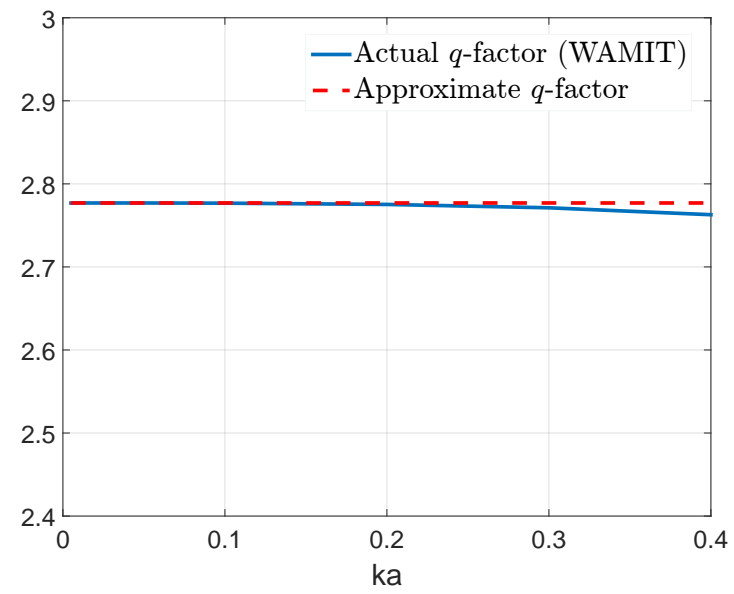

(a) Optimal Layout

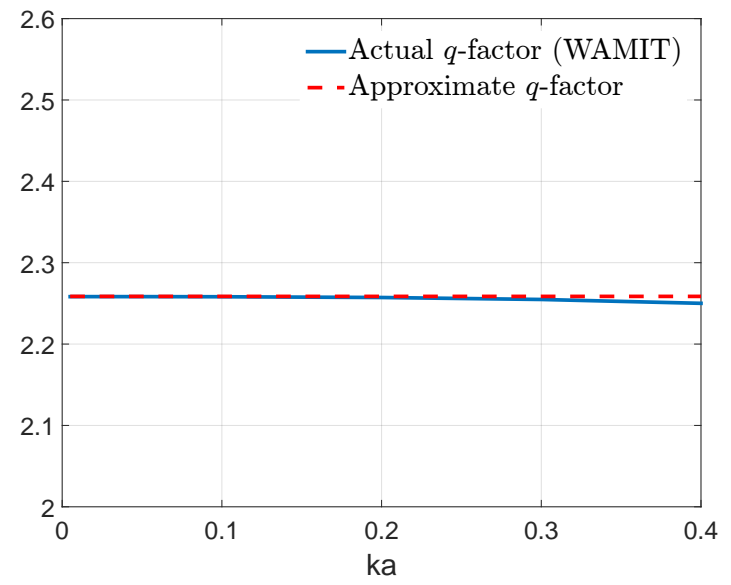

(b) Suboptimal Layout

Figure 5: Acuracy of point-absorber approximation

\section{Computational Results}

We begin with an experiment that confirms the accuracy of the point-absorber approximation. We used WAMIT (WAMIT, Inc. 2014) to compute the exact $q$-factor for two 5-WEC layouts, the best-known layout under the point-absorber approximation (Fitzgerald and Thomas 2007) and another, suboptimal, layout. Both layouts consist of identical cylindrical devices with a radius of $5 \mathrm{~m}$ and a height of $20 \mathrm{~m}$. In general, the accuracy of the point-absorber approximation depends on the radius of the WEC, $a$, relative to the wavenumber, $k$. Keeping $a$ fixed at 5 and varying $k$ between 0 and 0.08 , i.e., varying $k a$ between 0 and 0.4 , we calculated the exact $q$-factor (from WAMIT) and the $q$-factor under the point absorber approximation. The results for both layouts are plotted in Figure 5. The maximum error is approximately $0.5 \%$, and the average error is much smaller. As a point of comparison, Ocean Power Technologies' APB-350 and PB40 PowerBuoy models have radii of $1.35 \mathrm{~m}$ and $3.5 \mathrm{~m}$, respectively. A typical wavelength at ocean depths where the buoys would be deployed is greater than $100 \mathrm{~m}$. For these settings, we have $k a<0.09$ and 0.22 , respectively, and for these values the error is negligible.

Next, we investigate the performance of our two-phase heuristic. We tested our heuristic for $N=$ $3, \ldots, 15$. (Recall that an instance is completely specified by $N$ since the problem is isomorphic with respect to $\beta$ and $k$, by Proposition 1.) We are aware of very few other computational studies for the WE- 
CLP to use as benchmarks. Folley and Whittaker (2009) and Fitzgerald and Thomas (2007) consider $N=5$ only and report a solution with $q=2.777$, which we believe is optimal; and Mao (2013) provides results of his "tuned" genetic algorithm (GA) for $N \leq 15$. The results of Child (2011) and Child and Venugopal $(2009,2010)$ are not comparable to ours because they use a different method to calculate the $q$-factor and make different assumptions regarding the control mechanisms used by the WECs. Therefore, we compare our heuristic (with and without symmetry constraints) to Mao's (2013) tuned GA and with two general-purpose global optimization solvers, NOMAD (LeDigabel 2011) and PSWARM (Vaz and Vicente 2007). We conducted two sets of experiments, one using a 1-hour time limit and the other using a 24-hour time limit, for all algorithms. All algorithms are implemented and tested in MATLAB (R2014a) on a PC desktop with 2.10GHz x86 64-bit Intel(R) Core(TM)2 Duo CPU and 4.00 GB RAM.

Tables 2 and 3 and Figures 7(a) and 7(b) present the results of the 1- and 24-hour experiments, respectively. They list the best reported $q$-factor for $N=3, \ldots, 15$ for our heuristic and the other three algorithms. In the tables, the maximum $q$-factor found by any approach for a given $N$ is indicated in boldface.

Our heuristic finds the the best known solution from the literature for $N=5$ (Fitzgerald and Thomas 2007, Folley and Whittaker 2009) and consistently dominates NOMAD and PSWARM for both 1- and 24-hour time limits. It outperforms the tuned GA by Mao (2013) for all instances except $N=8$ and $N=11$ in the 1-hour experiment and for $N=12$ and $N=13$ in the 24-hour experiment. The two-phase heuristic with symmetry constraints tends to outperform the heuristic without, especially for the longer run time. The gap between our heuristics and the others tends to increase as $N$ increases. These results highlight the importance of exploiting properties of the solution structure in the solution approach to the WECLP, as our heuristic, and to a lesser extent that of Mao (2013), do.

It is worth noting that the results in the " $q$ [WOSymC]" column are not necessarily asymmetric, but rather that the local optimization phase does not enforce the symmetry constraint explicitly. Also, the instances for which the WOSymC heuristic finds a better solution than WSymC (e.g., $N=9,10$, and 15 in Table 2) do not necessarily disprove Conjecture 1, since WSymC may not have found the optimal solution.

These results provide insights that help guide the design of wave farms. First, from Figure 6, we observe that the best layouts are symmetric with respect to the wave direction, except for the layout with $N=4$ devices (which may not be optimal). Second, if we reflect any of the layouts about a line normal to the wave heading, we get the same $q$-factor, a result supported by Fitzgerald and Thomas's 2007 proof that $q(\beta+\pi)=q(\beta)$. Third, the computational results show that for a fixed region, as the number of devices increases, the performance of the farm does not increase proportionally, and furthermore, after some point, adding more devices degrades the performance. For example, in Figure 7(b), the farm with 
$N=7$ devices performs better compared to the other farms. (It is true in theory that for sufficiently large $N$, the $q$-factor begins to decrease or the problem becomes infeasible. Whether this is the effect observed in Figure 7(b) or whether our heuristic is simply not finding optimal solutions for the larger problems is impossible to tell.) Therefore, for a fixed region, determining the optimum number of devices in the farm is important due to the high cost of each device.

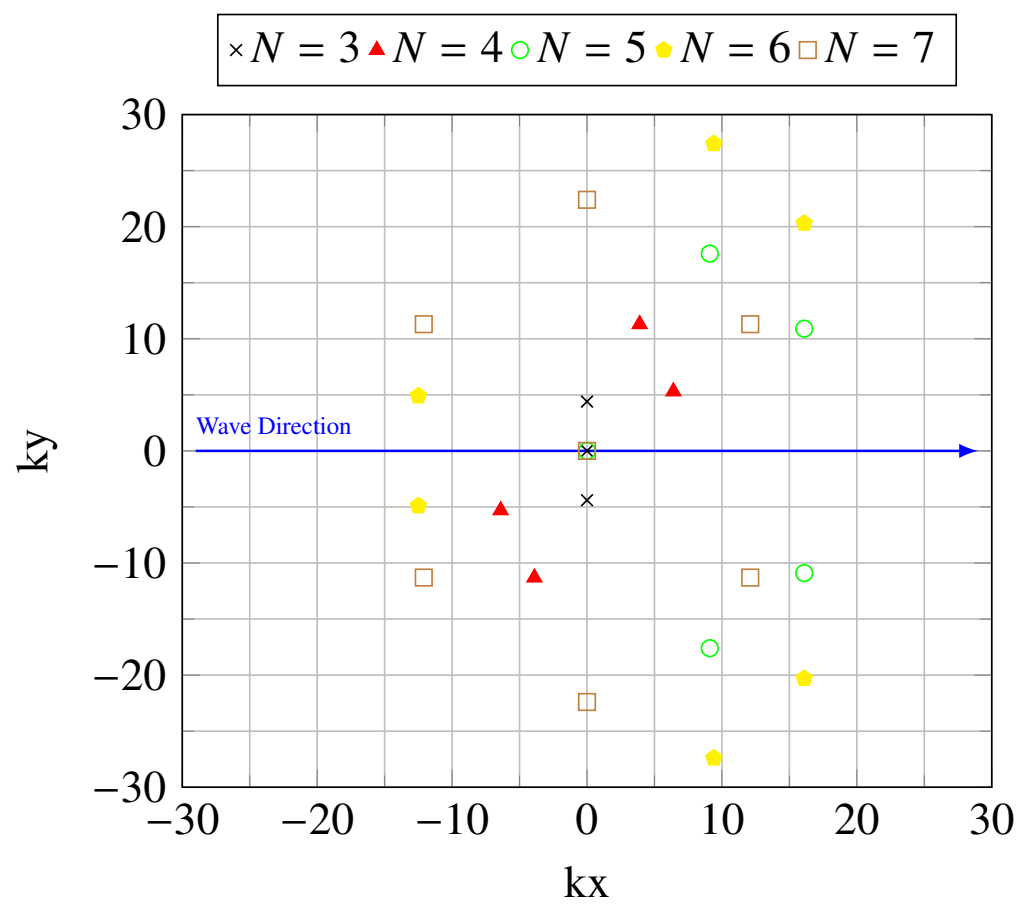

Figure 6: Best Known Layouts for Farms with $N=3, \ldots, 7$ (all layouts have a device at the origin)

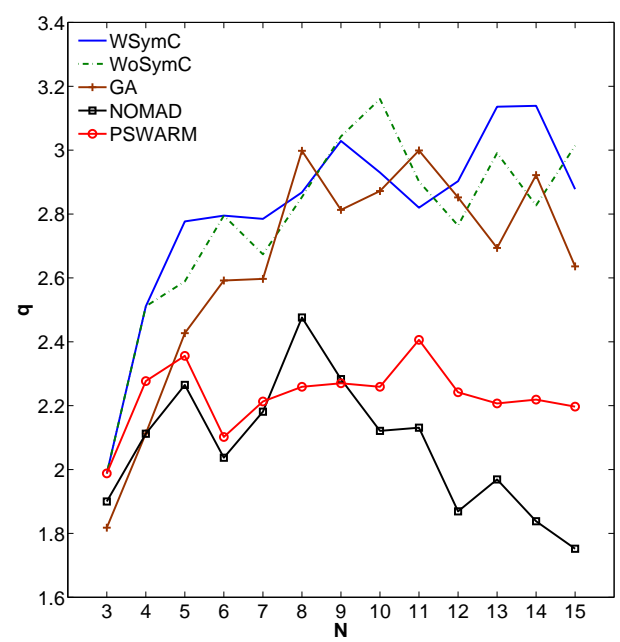

(a) 1-hour run time

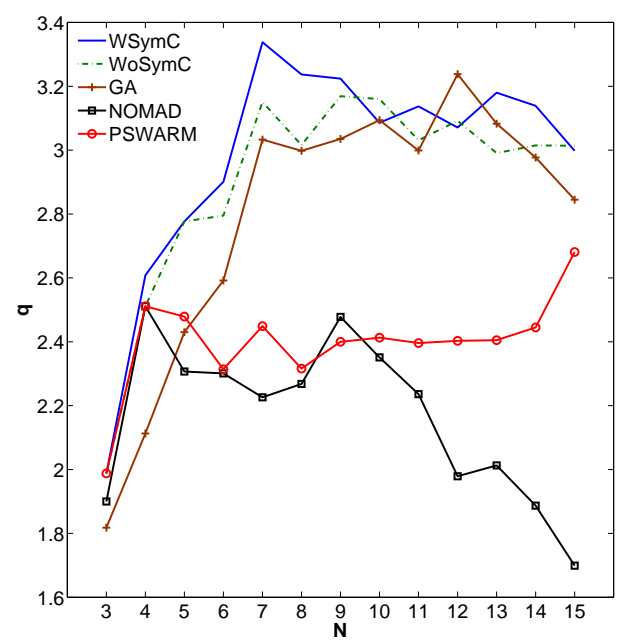

(b) 24-hour run time

Figure 7: Best $q$-Factor Obtained by Heuristics 


\begin{tabular}{|c|ccccc|}
\hline$N$ & $q[$ WSymC $]$ & $q[$ WOSymC $]$ & $q[\mathrm{GA}]$ & $q$ [NOMAD $]$ & $q$ [PSWARM] \\
\hline 3 & $\mathbf{1 . 9 8 8}$ & $\mathbf{1 . 9 8 8}$ & 1.818 & 1.900 & $\mathbf{1 . 9 8 8}$ \\
4 & $\mathbf{2 . 5 1 1}$ & $\mathbf{2 . 5 1 1}$ & 2.113 & 2.112 & 2.277 \\
5 & $\mathbf{2 . 7 7 7}$ & 2.589 & 2.427 & 2.265 & 2.356 \\
6 & $\mathbf{2 . 7 9 5}$ & $\mathbf{2 . 7 9 5}$ & 2.592 & 2.037 & 2.102 \\
7 & $\mathbf{2 . 7 8 5}$ & 2.674 & 2.597 & 2.181 & 2.213 \\
8 & 2.868 & 2.853 & $\mathbf{2 . 9 9 8}$ & 2.476 & 2.259 \\
9 & 3.029 & $\mathbf{3 . 0 4 3}$ & 2.813 & 2.283 & 2.270 \\
10 & 2.930 & $\mathbf{3 . 1 6 0}$ & 2.872 & 2.121 & 2.259 \\
11 & 2.820 & 2.902 & $\mathbf{2 . 9 9 9}$ & 2.131 & 2.406 \\
12 & $\mathbf{2 . 9 0 3}$ & 2.764 & 2.852 & 1.869 & 2.242 \\
13 & $\mathbf{3 . 1 3 6}$ & 2.991 & 2.694 & 1.969 & 2.207 \\
14 & $\mathbf{3 . 1 3 9}$ & 2.827 & 2.922 & 1.838 & 2.219 \\
15 & 2.878 & $\mathbf{3 . 0 1 4}$ & 2.636 & 1.752 & 2.197 \\
\hline
\end{tabular}

Table 2: Results of Two-Phase Heuristic vs. GA, NOMAD and PSWARM for $T=1$ (hr) 


\begin{tabular}{|c|ccccc|}
\hline$N$ & $q[$ WSymC $]$ & $q[$ WOSymC $]$ & $q[\mathrm{GA}]$ & $q$ [NOMAD $]$ & $q$ [PSWARM] \\
\hline 3 & $\mathbf{1 . 9 8 8}$ & $\mathbf{1 . 9 8 8}$ & 1.818 & 1.900 & $\mathbf{1 . 9 8 8}$ \\
4 & $\mathbf{2 . 6 0 8}$ & 2.511 & 2.113 & 2.511 & 2.511 \\
5 & $\mathbf{2 . 7 7 7}$ & $\mathbf{2 . 7 7 7}$ & 2.430 & 2.307 & 2.479 \\
6 & $\mathbf{2 . 9 0 1}$ & 2.795 & 2.592 & 2.301 & 2.314 \\
7 & $\mathbf{3 . 3 3 8}$ & 3.151 & 3.033 & 2.226 & 2.449 \\
8 & $\mathbf{3 . 2 3 7}$ & 3.016 & 2.998 & 2.268 & 2.316 \\
9 & $\mathbf{3 . 2 2 4}$ & 3.169 & 3.035 & 2.478 & 2.400 \\
10 & 3.086 & $\mathbf{3 . 1 6 0}$ & 3.094 & 2.351 & 2.413 \\
11 & $\mathbf{3 . 1 3 7}$ & 3.031 & 2.999 & 2.236 & 2.396 \\
12 & 3.071 & 3.092 & $\mathbf{3 . 2 3 8}$ & 1.979 & 2.403 \\
13 & $\mathbf{3 . 1 8 0}$ & 2.991 & 3.083 & 2.013 & 2.405 \\
14 & $\mathbf{3 . 1 3 9}$ & 3.015 & 2.977 & 1.887 & 2.445 \\
15 & 2.998 & $\mathbf{3 . 0 1 4}$ & 2.845 & 1.699 & 2.401 \\
\hline
\end{tabular}

Table 3: Results of Two-Phase Heuristic vs. GA, NOMAD and PSWARM for $T=24$ (hr)

\section{Conclusion}

In this work, we study optimization models and algorithms for the optimal configuration of wave farms. We develop theoretical properties for the $q$-factor under the point-absorber approximation and derived an analytical optimal solution for the 2-WEC problem. The analytical solution for the 2-WEC problem provides insights into the relative spacing among the WECs in near-optimal solutions. Based on these insights, we propose a heuristic optimization algorithm for choosing layouts to maximize the $q$-factor. Our results demonstrate that the heuristic generally outperforms the WECLP-specific genetic algorithm by Mao (2013) and the general-purpose global solvers PSWARM and NOMAD.

Our heuristic does not exploit our result that the WECLP problem is isomorphic. One possible method for improving a given solution would be to take the solution, find the $\beta$ and/or $k$ for which the solution gives the greatest $q$-factor, and then rotate and/or scale the resulting layout to reconfigure it for the original $\beta$ and $k$. The local optimization step could be added, as well. Developing a more efficient algorithm for finding good master layouts is another possible avenue for future research.

The methods and analysis described in this paper are based on deterministic, simplified ocean states. Real ocean waves have multiple sinusoidal components (rather than the single component assumed 
here), and our work can and should be extended to accommodate a more realistic model of the ocean waves and the resulting hydrodynamics. Moreover, ocean waves are stochastic, and these uncertainties can have a substantial degrading effect on the power of the wave farm (Snyder and Moarefdoost 2014). Snyder and Moarefdoost (2015) study models with stochastic wave direction $\beta$, but still assume simple waves with a single sinusoidal component. Exploring the WECLP in more realistic sea environments with irregular (multi-component) waves to is an important avenue for future research.

\section{Acknowledgments}

This research was supported in part by NSF grants CMMI-1400164 and CCF-1442858, and by a Lehigh University Accelerator grant. This support is gratefully acknowledged.

\section{References}

I. Borg and P. Groenen. Modern multidimensional scaling: Theory and applications. Springer, 2005.

K. Budal. Theory for absorption of wave power by a system of interacting bodies. Journal of Ship Research, 21(4):248-258, 1977.

B. Child. On the configuration of arrays of floating wave energy converters. PhD thesis, The University of Edinburgh, 2011.

B. Child and V. Venugopal. Modification of power characteristics in an array of floating wave energy devices. In Proceedings of the 8th European Wave and Tidal Energy Conference, Uppsala, Sweden, pages 309-318, 2009.

B. Child and V. Venugopal. Optimal configurations of wave energy device arrays. Ocean Engineering, $37: 1402-1417,2010$.

EPRI. Mapping and assessment of the united states ocean wave energy resource. Electric Power Research Institute, 2011. URL http://www1.eere.energy.gov/water//pdfs/ mappingandassessment.pdf.

J. Falnes. Radiation impedance matrix and optimum power absorption for interacting oscillators in surface waves. Applied Ocean Research, 2(2), 1980.

C. Fitzgerald and G. Thomas. A preliminary study on the optimal formation of an array of wave devices. In The 7th European Wave and Tidal Energy Conference, Porto, Portugal, 2007. 
M. Folley and T. Whittaker. The effect of sub-optimal control and the spectral wave climate on the performance of wave energy converter arrays. Applied Ocean Research, 31:260-266, 2009.

S. Kishore, L. Snyder, and P. Pradhan. Electricity from ocean wave energy: Technologies, opportunities and challenges. IEEE Smart Grid Newsletter, February 2013.

S. LeDigabel. Algorithm 909: Nomad: Nonlinear optimization with the mads algorithm. ACM Transactions on Mathematical Software (TOMS), 37(4):44, 2011.

L. Liberti, C. Lavor, N. Maculan, and A. Mucherino. Euclidean distance geometry and applications. ArXiv e-prints, 2012.

I. López and G. Iglesias. Efficiency of owc wave energy converters: A virtual laboratory. Applied Ocean Research, 44:63-70, 2014.

L. Mao. Optimizing wave farm layouts under uncertainty. Msc thesis, Lehigh University, 2013.

S. Mavrakos and P. McIver. Comparison of methods for computing hydrodynamic characteristics of arrays of wave power devices. Applied Ocean Research, 19(5):283-291, 1997.

P. McIver. Some hydrodynamic aspects of arrays of wave energy devices. Applied Ocean Research, 16: 61-69, 1994.

C. Mei. The Applied Dynamics of Ocean Surface Waves. Advanced series on ocean engineering. World Scientific, 1989. ISBN 9789971507893. URL https://books.google.com/books?id= awL1nQEACAAJ.

R. Parhizkar. Euclidean Distance Matrices: Properties, Algorithms and Applications. PhD thesis, ÉCOLE POLYTECHNIQUE FÉDÉRALE DE LAUSANNE, 2013.

I. Schoenberg. Remarks to maurice frechet's article: "sur la definition axiomatique d'une classe d'espace distances vectoriellement applicable sur l'espace de hilbert". Annals of Mathematics, pages 724-732, 1935.

L. Snyder and M. Moarefdoost. Layouts for ocean wave energy farms: Models, properties, and heuristic. In The 2nd Marine Energy Technology Symposium (METS), 2014.

L. Snyder and M. Moarefdoost. Optimizing wave farm layouts under uncertainity. In The 3rd Marine Energy Technology Symposium (METS), 2015.

A.J. Surrey and William Page. Some issues in the current debate about energy and natural resources. Omega, 2(5):651-665, 1974. 
G. Thomas and D. Evans. Arrays of three dimensional wave energy absorbers. Journal of Fluid Mechanics, 108:67-88, 1981.

I. Vaz and L. Vicente. A particle swarm pattern search method for bound constrained global optimization. Journal of Global Optimization, 39(2):197-219, 2007.

WAMIT, Inc., 2014. URL http://wamit. com.

Yi-Ming Wei, Zhi-Fu Mi, and Zhimin Huang. Climate policy modeling: An online sci-e and \{SSCI\} based literature review. Omega, 57, Part A:70-84, 2015. 